\title{
OPTIMIZATION OF A CONTINUOUS PULSED CORONA REACTOR
}

\author{
B. Srinivasan ${ }^{\ddagger}$, Srinivas Palanki ${ }^{\dagger}$, \\ David Grymonpré ${ }^{\dagger}$, and Bruce R. Locke ${ }^{\dagger}$ \\ ${ }^{\dagger}$ Department of Chemical Engineering, \\ Florida A\&M University-Florida State University, \\ Tallahassee, FL, 32310-6046. \\ $\ddagger$ Institut d’Automatique \\ École Polytechnique Fédérale de Lausanne \\ CH-1015 Lausanne, Switzerland
}

January 28, 2000

\begin{abstract}
Pulsed corona discharge processes utilize chemical radicals and ions and highly reactive molecules produced from a high voltage pulsed electrical discharge that is sustained in an aqueous phase medium. Recently, such processes have been used for treating organic contaminants from wastewater. The principle objective of this work is to optimize the hydrogen peroxide needed in the flow reactor to degrade phenol to a prespecified level with respect to the chemistry induced by the pulsed streamer corona. A simplified model of the corona reactor is utilized in conjunction with dynamic optimization and scaling analysis is used to derive analytical expressions for calculating the optimal hydrogen peroxide concentration. Simulations are performed to verify the performance of this optimization strategy.
\end{abstract}

\section{Introduction}

There is a continuing need for the development of efficient and cost effective processes to remove organic contaminants from groundwater and wastewater. In the process of removing waste contaminants it is also desirable to degrade them to harmless products. Advanced oxidation processes (AOT's) are a class of processes that degrade (usually though oxidation pathways) a range of organic contaminants. Over the past decade, an advanced oxidation technology that utilizes a pulsed streamer corona discharge in the liquid phase (Clements et al., 1985; Sharma et al., 1993; Joshi et al., 1995; Goheen et al., 1992) has been found to be effective at removing and degrading organic contaminants from aqueous solutions.

Pulsed corona discharge processes utilize chemical radicals and ions (e.g. $e_{a q}^{-}, H O^{-}, H^{*}, H O_{2}^{\cdot}, O^{1}(D)$ ) and highly reactive molecules, (e.g., $\mathrm{O}_{3}, \mathrm{H}_{2} \mathrm{O}_{2}$ ) produced from a high voltage pulsed electrical discharge that is sustained in an aqueous phase medium. Preliminary studies have demonstrated the complete degradation of phenol in solution (Sharma, 1993; Sharma et al., 1993) and the production of ozone when oxygen or air is sparged through the needle electrodes immersed in the water (Clements et al., 1985). The combination of activated carbon particles with the pulsed corona discharge also leads to enhanced power efficiency and possible surface-phase induced catalytic chemical reactions (Grymonpré, 1998; Grymonpré, et al., 1998).

Initial work has only been done in batch reactors, with one single corona discharge. However, for large-scale applications, such a reactor would be inadequate and a different reactor configuration is necessary. One such 
reactor configuration is a flow reactor which would allow for a contaminated water sample to flow continuously through the corona region, thereby increasing the amount of sample that can be treated.

The principle objective of this work is to optimize the hydrogen peroxide needed in the flow reactor to degrade the phenol to a prespecified level with respect to the chemistry induced by the pulsed streamer corona. This will involve using a simplified model of the phenol degradation in combination with dynamic optimization. It will be shown that the optimum solution is on a constraint and analytical expressions for the input can be obtained. The case where the inlet phenol concentraion is varying will be considered and two types of solution will be proposed depending on whether or not the measurement of the phenol concentraion is available.

\section{Model Formulation}

Initial models of the bulk phase corona induced chemical reactions were reported by Joshi et al. (1993). In that study it was assumed that the pulsed corona discharge leads to the formation of hydrogen peroxide, hydroxyl radicals, and aqueous electrons. Of these reactive species, the hydroxyl radical is the dominant reactive species, but quickly recombines to form hydrogen peroxide. In combination with ferrous ions $\left(F e^{2+}\right)$, hydrogen peroxide reacts to form hydroxyl radicals in the bulk solution. For this reason, the present model will predict hydrogen peroxide concentrations to optimize the oxidation products of phenol.

The reactions used in this model are given in Table 1 along with the reactions rate constants. The first two reactions are the Fenton reactions, which involve the production of hydroxyl radicals from hydrogen peroxide. The third reaction is the phenol oxidation reaction, which for this simplified model, will produce only oxidation products. The final three reactions are a few of the possible radical termination and combination reactions, which are well known from radiation chemistry (Buxton, 1987; Magee and Chatterjee, 1987). The rates of formation of hydrogen peroxide, hydroxyl radicals, and aqueous electrons have also been determined (Joshi, 1994; Joshi et al., 1995).

\begin{tabular}{|c|c|}
\hline $\begin{array}{l}\mathrm{Fe}^{2+}+\mathrm{H}_{2} \mathrm{O}_{2} \stackrel{k_{1}}{\rightarrow} \mathrm{Fe}^{3+}+\mathrm{OH}^{-}+\mathrm{HO}^{\cdot} \\
\mathrm{Fe}^{2+}+\mathrm{HO}^{\cdot} \stackrel{k_{2}}{\rightarrow} \mathrm{Fe}^{3+}+\mathrm{OH}^{-} \\
\text {Phenol }+\mathrm{HO}^{\cdot} \stackrel{k_{3}}{\rightarrow} \text { Products }+\mathrm{H}^{\cdot} \\
\mathrm{H}^{\cdot}+\mathrm{HO}^{\cdot} \stackrel{k_{4}}{\rightarrow} \mathrm{H}_{2} \mathrm{O} \\
2 \mathrm{HO} \stackrel{\mathrm{k}_{5}}{\rightarrow} \mathrm{H}_{2} \mathrm{O}_{2} \\
\mathrm{H}^{\cdot}+\mathrm{H}_{2} \mathrm{O}_{2} \stackrel{k_{6}}{\rightarrow} \mathrm{H}_{2} \mathrm{O}+\mathrm{HO}\end{array}$ & $\begin{aligned} k_{1} & =76 M^{-1} s^{-1} \\
k_{2} & =5 \times 10^{8} M^{-1} s^{-1} \\
k_{3} & =6.5 \times 10^{9} M^{-1} s^{-1} \\
k_{4} & =2.4 \times 10^{10} M^{-1} s^{-1} \\
k_{5} & =4 \times 10^{9} M^{-1} s^{-1} \\
k_{6} & =1.0 \times 10^{10} M^{-1} s^{-1}\end{aligned}$ \\
\hline
\end{tabular}

The general material balance for a plug-flow reactor is

$$
\frac{d c_{i}}{d V}=\frac{1}{\nu} r_{i}
$$

where $c_{i}$ is the molar concentration of the $i^{t h}$ species, $V$ is the volume of the reactor, $\nu$ is the volumetric flow rate, and $r_{i}$ is the reaction rate of the $i^{t h}$ species. Applying this equation to the chemical reactions given in Table 
1, the following equations arise

$$
\begin{aligned}
\frac{d x_{1}}{d V} & =\frac{1}{\nu}\left[-k_{3} x_{1} x_{2}\right] \\
\frac{d x_{2}}{d V} & =\frac{1}{\nu}\left[k_{1} x_{4} u-k_{2} x_{2} x_{4}-k_{3} x_{1} x_{2}-k_{4} x_{2} x_{6}-2 k_{5} x_{2}^{2}+k_{6} x_{6} u\right] \\
\frac{d x_{3}}{d V} & =\frac{1}{\nu}\left[k_{3} x_{1} x_{2}\right] \\
\frac{d x_{4}}{d V} & =\frac{1}{\nu}\left[-k_{1} x_{4} u-k_{2} x_{2} x_{4}\right] \\
\frac{d x_{5}}{d V} & =\frac{1}{\nu}\left[k_{1} x_{4} u+k_{2} x_{2} x_{4}\right] \\
\frac{d x_{6}}{d V} & =\frac{1}{\nu}\left[k_{3} x_{1} x_{2}-k_{4} x_{2} x_{-} k_{6} x_{6} u\right]
\end{aligned}
$$

where $x_{1}=[$ Phenol $], x_{2}=\left[\mathrm{HO}^{\cdot}\right], x_{3}=[$ Products $], x_{4}=\left[\mathrm{Fe}^{2+}\right], x_{5}=\left[\mathrm{OH}^{-}\right], x_{6}=\left[\mathrm{H}^{\cdot}\right]$, and $u=\left[\mathrm{H}_{2} \mathrm{O}_{2}\right]$. The objective is to determine the concentration of hydrogen peroxide along the length of the reactor to degrade phenol to a prespecified level at the end of the reactor.

The concentration of phenol at the inlet varies between $0.9 \times 10^{-3} \mathrm{~mol} / \mathrm{L}$ and $1.1 \times 10^{-3} \mathrm{~mol} / \mathrm{L}$ for a typical waste stream considered in this paper. The concentration of the ferrous ions at the reactor inlet is assumed to be $4.85 \times 10^{-4} \mathrm{~mol} / \mathrm{L}$. The concentrations of all other species is assumed to be equal to zero at the reactor inlet. The volumetric flow rate $\nu=1 \mathrm{~cm}^{3} / \mathrm{s}$ and the length of the reactor is such that the exit volume $V_{\max }=10 \mathrm{~cm}^{3}$.

\section{Reactor Optimization}

To run the reactor optimally, it is necessary to calculate the minimum amount of hydrogen peroxide necessary to bring the phenol concentration below a prespecified limit at the end of the reactor. In this section, this amount of hydrogen peroxide will be computed by formulating the problem as a dynamic optimization problem and by performing a simple time-scale analysis.

\subsection{Problem Formulation}

The optimization problem can be mathematically stated as follows:

$$
\min J=\int_{0}^{V_{\max }} u d V
$$

subject to the dynamic equations (2) - (7) and the terminal constraint:

$$
x_{1}\left(V_{\max }\right) \leq x_{\min }
$$

where $x_{\min }$ is the minimum allowable concentration of phenol in the exit stream of the reactor. This class of optimization problems can be solved, in general, using dynamic optimization methods which involve the solution of a two point boundary value problem (Bryson and Ho, 1975). Usually, such problems are computationally expensive to solve. However, we will show in the succeeding subsections, that the model equations (2) - (7) can be simplified considerably by doing a simple time-scale analysis. This simplification will result in a closed form solution for the evolution of phenol. Also, an analytical expression will be obtained for the case when the phenol concentration varies with time at the inlet of the reactor. These closed form solutions will be used to calculate the optimum hydrogen peroxide concentration necessary to degrade the phenol.

\subsection{Model Reduction}

The system of equations (2) - (7) can be simplified by noting the following facts. 
1. $x_{1}+x_{3}=$ constant and $x_{4}+x_{6}=$ constant. This allows us to remove $x_{3}$ and $x_{6}$ from the system of equations. Moreover, $x_{3}$ and $x_{6}$ do not appear in the reaction rates $r_{i}$ for any $i$.

2. Due to the difference in speed of various reactions, two of the states, $x_{2}$ amd $x_{7}$, are in pseudo-steady state with their values around $1 e^{-11}$. With this knowledge, $x_{2}$ and $x_{7}$ can be replaced as functions of other states and inputs.

It can be seen from the numerical values of different constants and states, $k_{4} x_{2} x_{7}$ is several orders of magnitude smaller than $k_{3} x_{1} x_{2}$ and $k_{6} x_{7} u$. Since $x_{7}$ is in pseudo steady state,

$$
\frac{d x_{7}}{d V}=0
$$

which implies

$$
k_{3} x_{1} x_{2}=k_{6} x_{7} u
$$

Substituting this in equation (3) and removing terms which are of the magnitude $1 e^{-10}$, we get,

$$
k_{1} x_{4} u=k_{2} x_{4} x_{2}
$$

Using the two relations, (11) and (12), we get

$$
x_{2}=\left(\frac{k_{1}}{k_{2}}\right) u \quad \text { and } \quad x_{7}=\frac{\left(k_{1} k_{3}\right)}{\left(k_{2} k_{6}\right)} x_{1}
$$

So, the system equations (2) - (7) are completely described by

$$
\begin{aligned}
& \frac{d x_{1}}{d V}=\left(\frac{-k_{3} k_{1}}{\nu k_{2}}\right) x_{1} u \\
& \frac{d x_{4}}{d V}=\left(\frac{-2 k_{1}}{\nu}\right) x_{4} u
\end{aligned}
$$

Also, unlike the system of equations (2)-(7), these (14)-(15) are completely decoupled and only the first one is related to the optimizing objective of reducing the concentration of phenol. Hence only the evolution of $x_{1}$ will be studied.

\subsection{Analytic expressions for Phenol Evolution}

An analytic expression for the solution of $x_{1}$ can be easily obtained by using the following length scaling. Let a new lenght scale $\tau$ be chosen such that $\frac{d \tau}{d V}=u$ implying $\tau=\int_{0}^{V} u(\mu) d \mu$. In this new length scale the system equations are

$$
\frac{d x_{1}}{d \tau}=-\alpha x_{1}
$$

where $\alpha=\left(\frac{k_{3} k_{1}}{\nu k_{2}}\right)$. The system is linear and homogeneous in the new length scale and the solution is given by,

$$
x_{1}(V)=x_{1}(0) e^{-\alpha \tau}=x_{1}(0) e^{-\alpha \int_{0}^{V} u(\mu) d \mu}
$$

\subsection{Optimal Hydrogen Peroxide Calculation}

The cost function for a given $x_{1}(0)$ is given

$$
J=\int_{0}^{V_{\max }} u(\mu) d \mu=\frac{1}{\alpha} \log \left(\frac{x_{1}(0)}{x_{1}\left(V_{\max }\right)}\right)
$$


This implies that larger the value of $x_{1}\left(V_{\max }\right)$, smaller is the cost. On the other hand, the constraint $x_{1}\left(V_{\max }\right) \leq$ $x_{\min }$ prevents the choice of a large $x_{1}\left(V_{\max }\right)$ and so the optimum is at $x_{1}\left(V_{\max }\right)=x_{\min }$. Hence

$$
J_{o p t}=\frac{1}{\alpha} \log \left(\frac{x_{1}(0)}{x_{\min }}\right)
$$

and the optimal input is any $u$ which guarentees $J_{\text {opt }}=\int_{0}^{V_{\max }} u(\mu) d \mu$. Note that the profile of the input is immaterial as long as the condition on the integral is satified. We hence choose a constant input

$$
u(V)=\frac{1}{\alpha V_{\max }} \log \left(\frac{x_{1}(0)}{x_{\min }}\right), \forall V
$$

to bring the phenol concentration to $x_{\min }$ at the end of the reactor.

\subsection{Time-varying Phenol inlet}

Next we will consider the fact that $x_{1}(0)$ can vary with time. This is typically the case in a waste stream where the contaminant concentration changes due to changes in upstream conditions. In such a case, $x_{1}$ will be a function of time and space and the analytical solution can be written as

$$
x_{1}(V, t)=x_{1}\left(0, t-\frac{V}{\nu}\right) e^{-\alpha \int_{0}^{V} u\left(\mu, t-\left(\frac{V-\mu}{\nu}\right)\right) d \mu}
$$

The above expression can be obtained by noting that the contents of the reactor move along the column at a speed of $1 / \nu$. In the absence of any reaction along the column,

$$
x_{1}(V, t)=x_{1}\left(0, t-\frac{V}{\nu}\right)
$$

The exponential term represents the consumption of phenol along the column as a function of the concentration of $\mathrm{H}_{2} \mathrm{O}_{2}$.

Despite the variation of the inlet phenol concentration, the optimum lies at $x_{1}\left(V_{\max }\right)=x_{\min }$. The optimum input $u(V, t)$ corresponding to keeping $x_{1}\left(V_{\max }\right)=x_{\min }$ is given by

$$
u(V, t)=\frac{1}{\alpha V_{\max }} \log \left(\frac{x_{1}\left(0, t-\frac{V}{\nu}\right)}{x_{\text {lim }}}\right)
$$

If $x_{1}(0, t)$ can be measured, then the above feedback law can be implemented. However, if the measurement of phenol concentration is not available, one has to take a conservative stand and use a value of $u$ which will guarentee that the outlet phenol concentration is less than $x_{\min }$ even for the maximum value of $x_{1}(0, t), x_{\max }$. Then the conservative constant optimal input is given by,

$$
u_{\text {conservative }}=\frac{1}{\alpha V_{\max }} \log \left(\frac{x_{\max }}{x_{\text {lim }}}\right), \forall V, \forall t
$$

The advantage of the conservative feedback is the simplicity - a constant for all values of space and time. The disadvantage, however, is that in the presence of large variations, one could do much better with the cost when measurements are available.

\subsection{Simulation Results}

Figure 1 shows the implementation of the conservative optimal input scheme and it is observed that the concentration of phenol at the outlet is indeed below the prespecified limit of $5 \times 10^{-5} \mathrm{~mol} / \mathrm{L}$. For $x_{\max }=1.1 \times 10^{-3}$, 
the conservative optimal input is given by $u_{\text {conservative }}=3.13 \times 10^{-4}$ constant for all time and space. This leads to a total consumption of $\mathrm{H}_{2} \mathrm{O}_{2}$ in $100 s, \int_{0}^{100} \mathrm{~J} d t=0.313$.

Figure 2 shows the implementation of the feedback scheme where an on-line measurement of the phenol concentration is available. In this case, the hydrogen peroxide concentration is adjusted using expression (23). It is observed that in this case, the phenol concentration at the reactor outlet is exactly at the prespecificed limit of $5 \times 10^{-5} \mathrm{~mol} / \mathrm{L}$. In this case the total consumption of $\mathrm{H}_{2} \mathrm{O}_{2}$ in $100 \mathrm{~s}, \int_{0}^{100} J d t=0.303$.

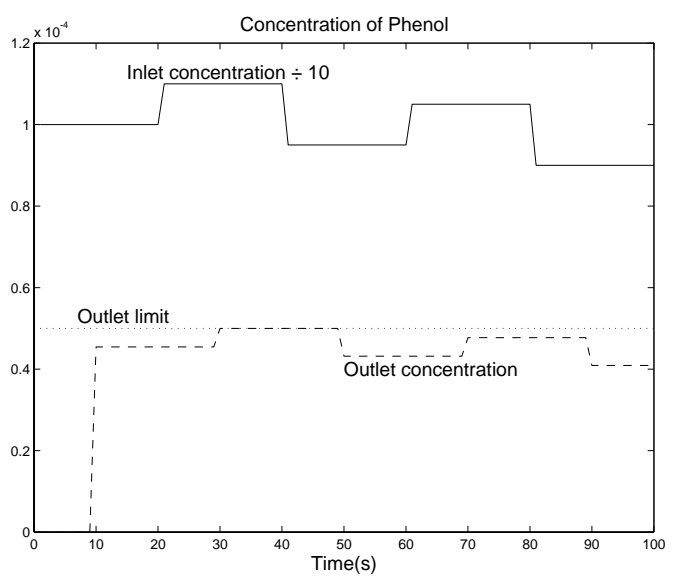

Figure 1: Conservative Optimization - constant $u$

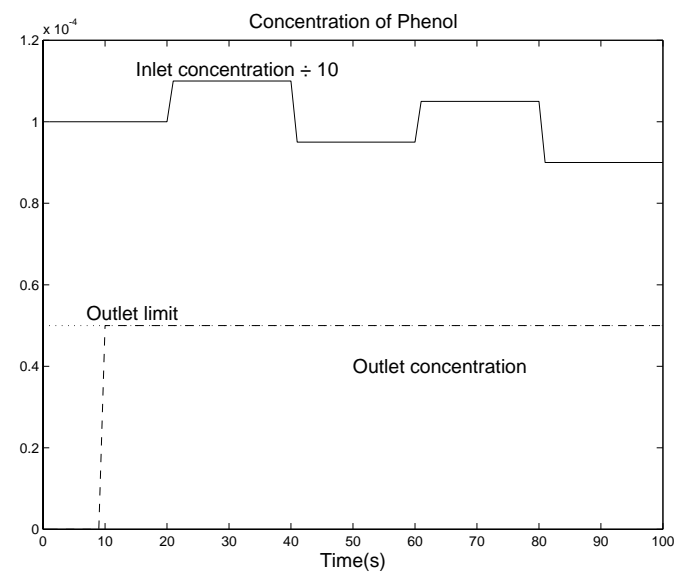

Figure 2: Optimization with Phenol Measurements - $u$ adjusted as in (23)

\section{Conclusions}

The minimum hydrogen peroxide concentration necessary to degrade phenol below a prespecified limit is calculated by considering a mathematical model of a pulsed corona reactor. The model described here is based on the chemistry of the pulsed corona. It is shown that the corona model can be simplified considerably by doing a simple scaling. This simplification permits the calculation of a closed form solution for the dynamic optimization problem. Simulations are performed to verify that the derived closed form solution results in the degradation of phenol to a level below a prespecified limit. It is shown that when a measurement of phenol is available at any point in the reactor, the concentration of hydrogen peroxide can be adjusted to guarantee that the concentration of phenol at the reactor outlet is below the prespecificed limit. In the absence of this on-line measurement, a conservative input strategy, based on the "worst case" level of phenol at the inlet, is presented. 


\section{References}

[1] Bryson, A.E., Ho, Y.C., 1975: Applied Optimal Control, Hemisphere Publishing Corp., New York, USA

[2] Clements, J.S., Sato, M., and Davis, R.H., 1985: Preliminary Investigation of Prebreakdown Phenomena and Chemical Reactions Using a Pulsed High Voltage Discharge in Water. IEEE Trans. Ind Appl. IA-23, p. 1372 .

[3] Gohenn, S. C., Durham, D. E., McCulloch, M., and Heath, W. O., 1992: The Degradation of Organic Dyes by Corona Discharge, Proceedings of the Second International Symposium Chemical Oxidation: Technology for the Nineties, p. 356.

[4] Grymonpre, D.R., 1998: The Effects of Carbon Particles on Aqueous Phase Pulsed Streamer Corona, M.S. Thesis, Florida State University, Tallahassee, FL.

[5] Grymonpre, D. R., W. C. Finney, and B. R. Locke, 1998: Activated Carbon Particles in Aqueous Phase Pulsed Streamer Corona Discharge, J. of Adv. Oxid. Tech., in press.

[6] Joshi, A.A., 1994: Formation of Hydroxyl Radicals, Hydrogen Peroxide, and Aqueous Electrons by Pulsed Streamer Corona Discharge in Aqueous Solutions, M.S. Thesis, Florida State University, Tallahassee, FL.

[7] Joshi, A.A., Locke, B.R., Arce, P., and Finney, W.C., 1995: Formation of Hydroxyl Radicals, Hydrogen Peroxide and Aqueous Electron by Pulsed Streamer Corona Discharge in Aqueous Solution, J. Hazardous Materials, 41(1) 3 (1995).

[8] Sharma, A. K., 1993: High Voltage Pulsed Streamer Corona Discharges for the Removal of Organic Contaminants From Aqueous Solutions, M.S.. Thesis, Florida A \& M University, Tallahassee, FL.

[9] Sharma, A. K., Locke, B. R., Arce, P., Finney, W. C., 1993, A Preliminary Study of Pulsed Streamer Corona Discharge for the Degradation of Phenol in Aqueous Solutions. Hazardous Waste 85 Hazardous Materials, 10 (2), p. 209. 